\title{
Impact of universal access to hepatitis C therapy on HIV-infected patients: implementation of the Spanish national hepatitis $\mathrm{C}$ strategy
}

\author{
A. Rivero-Juarez ${ }^{1}$ - L. F. Lopez-Cortes ${ }^{2} \cdot$ M. Castaño ${ }^{3}$ D. Merino ${ }^{4} \cdot$ M. Marquez $^{5}$. \\ M. Mancebo ${ }^{6}$ • F. Cuenca-Lopez ${ }^{1}$ • P. Jimenez-Aguilar ${ }^{7}$ • I. Lopez-Montesinos ${ }^{8}$ • \\ S. Lopez-Cardenas ${ }^{9}$ - A. Collado ${ }^{10}$ - M. A. Lopez-Ruz ${ }^{11} \cdot$ M. Omar $^{12} \cdot$ F. Tellez $^{13}$ • \\ X. Perez-Stachowski ${ }^{14}$ • J. Hernandez-Quero ${ }^{15}$ • J. A. Girón-Gonzalez ${ }^{16}$ • $^{\circ}$ \\ E. Fernandez-Fuertes ${ }^{17}$ - A. Rivero ${ }^{1}$ - on behalf of the HERACLES cohort study team of \\ the Grupo de Estudio de Hepatitis Virales (HEPAVIR) of the Sociedad Andaluza de \\ Enfermedades Infecciosas (SAEI)
}

Received: 5 August 2016/Accepted: 16 October 2016/Published online: 27 October 2016

(C) The Author(s) 2016. This article is published with open access at Springerlink.com

Abstract In April 2015, the Spanish National Health System (SNHS) developed a national strategic plan for the diagnosis, treatment, and management of hepatitis $\mathrm{C}$ virus (HCV). Our aim was to analyze the impact of this on human immunodeficiency virus (HIV)-infected patients included in the

A. Rivero-Juarez arjvet@gmail.com

A. Rivero ariveror@gmail.com

on behalf of the HERACLES cohort study team of the Grupo de Estudio de Hepatitis Virales (HEPAVIR) of the Sociedad Andaluza de Enfermedades Infecciosas (SAEI)

1 Unidad de Enfermedades Infecciosas, Hospital Universitario Reina Sofía de Córdoba, Instituto Maimonides de Investigación Biomédica de Córdoba (IMIBIC), Universidad de Córdoba (UCO), Avenida Menéndez Pidal s/n, 14004 Córdoba, Spain

2 Unidad Clínica de Enfermedades Infecciosas, Microbiología y Medicina Preventiva, Instituto de Biomedicina de Sevilla (IBiS), Hospital Universitario Virgen del Rocío/CSIC/Universidad de Sevilla, Seville, Spain

3 Unidad Clínica de Enfermedades Infecciosas, Hospital Regional Universitario Carlos Haya, Málaga, Spain

4 Unidad Clínica de Enfermedades Infecciosas, Complejo Hospitalario Universitario de Huelva, Huelva, Spain

5 Unidad Clínica de Enfermedades Infecciosas y Microbiología, Hospital Regional Universitario Virgen de la Victoria, Málaga, Spain
HERACLES cohort during the first 6 months of its implementation. The HERACLES cohort (NCT02511496) was set up in March 2015 to evaluate the status and follow-up of chronic HCV infection in patients co-infected with HIV in the south of Spain. In September 2015, the data were analyzed to identify

6 Unidad Clínica de Enfermedades Infecciosas y Microbiología, Instituto de Biomedicina de Sevilla (IBiS), Hospital Universitario de Valme, Seville, Spain

7 Unidad Clínica de Enfermedades Infecciosas, Hospital Universitario Puerto Real, Cádiz, Spain

8 Unidad Clínica de Enfermedades Infecciosas y Microbiología, Hospital Universitario Virgen Macarena, Seville, Spain

9 Unidad Clínica de Enfermedades Infecciosas y Microbiología, Hospital de Jerez, Jerez, Spain

10 Unidad Clínica de Enfermedades Infecciosas, Complejo Hospitalario Torrecárdenas, Almería, Spain

11 Unidad Clínica de Enfermedades Infecciosas y Microbiología, Hospital Universitario Virgen de las Nieves, Granada, Spain

12 Unidad Clínica de Enfermedades Infecciosas, Complejo Hospitalario de Jaén, Jaén, Spain

13 Unidad Clínica de Enfermedades Infecciosas y Microbiología, Hospital La Línea, AGS Campo de Gibraltad, Cádiz, Spain

14 Unidad de Medicina Interna, Hospital Costa del Sol, Marbella, Spain

15 Unidad Clínica de Enfermedades Infecciosas, Hospital Universitario San Cecilio, Granada, Spain

16 Unidad Clínica de Enfermedades Infecciosas y Microbiología, Hospital Puerta del Mar, Cádiz, Spain

17 Unidad de Medicina Tropical, Hospital de Poniente, El Ejido, Spain 
clinical events (death, liver decompensation, and liver fibrosis progression) and rate of treatment implementation in this population. The study population comprised a total of $3474 \mathrm{HIV} /$ $\mathrm{HCV}$ co-infected patients. The distribution according to liver fibrosis stage was: $1152 \mathrm{~F} 0-\mathrm{F} 1$ (33.2 \%); $513 \mathrm{~F} 2$ (14.4\%); 641 F3 (18.2 \%); 761 F4 (21.9 \%); and 407 whose liver fibrosis was not measured (12.3\%). During follow-up, 248 patients progressed by at least one fibrosis stage $[7.1 \% ; 95 \%$ confidence interval (CI): 6.3-8\%]. Among cirrhotic patients, 52 (6.8\%; $95 \%$ CI: 5.2-8.9\%) developed hepatic decompensation. In the overall population, 50 patients died $(1.4 \%$; $95 \%$ CI: $1.1-1.9 \%)$. Eight hundred and nineteen patients $(23.56 \%)$ initiated interferon (IFN)-free treatment during follow-up, of which $47.8 \%$ were cirrhotic. In our study, during 6 months of follow-up, $23.56 \%$ of HIV/HCV co-infected patients included in our cohort received HCV treatment. However, we observed a high incidence of negative short-term outcomes in our population.

\section{Introduction}

The high efficacy and safety of direct-acting antiviral drugs (DAAs) used for the treatment of hepatitis $\mathrm{C}$ virus (HCV) has meant a significant improvement in cure rates [defined as sustained virological response (SVR)] and, as a direct result, an improved prognosis for this infectious disease [1-3]. Nevertheless, incorporating DAAs into patient care requires implementing new and expensive resources, so limiting their application in countries like Spain, which have universal free healthcare systems. In this regard, in April 2015, the Spanish National Health System (SNHS) developed a strategic plan for diagnosing, treating, and managing $\mathrm{HCV}$ throughout Spain [4]. This plan was approved in April 2015 and extended across four strategic action lines, each with different objectives and key action points. These lines included: (i) quantifying the magnitude of this disease; (ii) defining the clinical criteria for implementing appropriate treatment; (iii) establishing coordination mechanisms to guarantee the proper implementation of the plan; and (iv) promoting knowledge about the prevention, diagnosis, and treatment of HCV by fomenting clinical and basic research [4]. To support these lines, key clinical criteria for starting HCV therapy were determined, as a result of which implementation of therapy was obligatory for the majority of patients (Table 1). The development and implementation of this plan, therefore, constitutes a major advance in the eradication of HCV in Spain.

HCV patients co-infected with human immunodeficiency virus (HIV) form an especially sensitive population. The use of highly active antiretroviral therapy (HAART) led to liver disease, mainly $\mathrm{HCV}$ infection, being identified as the main cause of morbidity and mortality among HIV-infected patients [5]. This effect is a direct consequence of higher and faster
Table 1 Criteria for treatment initiation and prioritization of the Spanish national strategic plan for the diagnosis, treatment, and management of $\mathrm{HCV}$ infection

Prioritization criteria for implementing HCV treatment

- All patients with liver fibrosis stages F4, F3, or F2

- Patients on the waiting list for liver transplant

- Patients with liver transplant, independent of stage of liver fibrosis

- Patients with failed treatment with Peg-IFN/RBV in combination with boceprevir or telaprevir

- Patient who are recipients of non-liver transplants, independent of stage of liver fibrosis

- Patients with extra-hepatic manifestations, independent of stage of liver fibrosis

- Patients with stage F0-F2 liver fibrosis, in the following situations:

- Patients at risk of transmission

- Women wishing to become pregnant

$H C V$ hepatitis $\mathrm{C}$ virus; $F 4$ liver cirrhosis; $F 3$ liver fibrosis stage $3 ; F 2$ liver fibrosis stage $2 ; F 1$ liver fibrosis stage $1 ; F 0$ absence of liver fibrosis; $P e g-I F N / R B V$ pegylated interferon plus ribavirin

liver fibrosis progression rates and lower sustained virological response (SVR) rates, even using interferon (IFN)-free treatments [6-8]. For these reasons, it is vitally important for this population of patients to be part of a national strategy.

There are very few reports showing the impact of a national program for treating chronic hepatitis $\mathrm{C}$ in $\mathrm{HIV} / \mathrm{HCV}$ coinfected patients. Here, therefore, we analyzed the impact on HIV-infected patients included in the HERACLES cohort of the Spanish national strategic plan for diagnosing, treating and managing $\mathrm{HCV}$ infection during the first 6 months of its implementation.

\section{Methods}

\section{Study design and population}

The HERACLES cohort, "Status of Chronic Liver Disease in Hepatitis C Virus (HCV) Patients Coinfected With Human Immunodeficiency Virus (HIV) in Andalusia", is a multicenter prospective observational cohort study of HIV-infected patients with active HCV co-infection (clinicaltrials.gov identification: NCT02511496). The cohort was set up in March 2015, with the main objective of evaluating the current status and follow-up of chronic HCV infection in patients coinfected with HIV in Andalusia (southern Spain).

This cohort included HIV-infected patients with chronic $\mathrm{HCV}$ infection in follow-up at 19 reference centers in Andalusia for the care of HIV-infected patients. These 19 centers look after a population of 15,556 HIV-infected patients, who represent $99.3 \%$ of HIV-infected patients attending 
centers in the Andalusia healthcare system. These data were obtained after an epidemiological inquiry in which all HIVinfected patients attending the 21 reference centers of Andalusia $(n=15,663)$ were tested [9]. HIV-infected patients were included in the cohort if they matched the following inclusion criteria: (i) active chronic $\mathrm{HCV}$ infection (defined as detectable HCV RNA in serum) and (ii) not receiving treatment for $\mathrm{HCV}$ at inclusion.

\section{Variable collection and definition}

At inclusion in the cohort (March-April 2015), the demographic, clinical, and virological characteristics of participants were collected and recorded. These were considered the baseline characteristics, and included: age, gender, risk for $\mathrm{HCV}$ infection, use of HAART, CD4+ cell count (cells/mL), plasma HIV viral load (copies/mL), acquired immune deficiency syndrome (AIDS)-defining criteria, HCV plasma viral load (IU/ $\mathrm{mL}$ ), HCV genotype/subtype, liver fibrosis stage, ChildTurcotte-Pugh (CTP) classification (A, B, or C), and history of $\mathrm{HCV}$ therapy.

Bi-annual follow-up of patients included in the cohort was planned. Variables collected and recorded at each follow-up visit included: liver fibrosis stage, use of DAA-based antiHCV therapy during follow-up, achieving SVR, presence and type of liver decompensation, inclusion on the waiting list for transplant, liver transplant, death, and cause of death. Variables for this report were collected in August-September 2015 (6-month follow-up).

Liver fibrosis staging was performed by liver biopsy (following the METAVIR fibrosis score) and/or liver transient elastography (FibroScan; Echosens, Paris, France). Classification of liver fibrosis staging was by histological analysis or liver stiffness measurement (LSM) score as follows: (i) F0-F1 METAVIR fibrosis score or LSM $<7.2$ $\mathrm{kPa}$; (ii) F2 METAVIR fibrosis score or LSM 7.2-8.9 kPa; (iii) F3 METAVIR fibrosis score or LSM 9-14.5; and (iv) F4 METAVIR fibrosis score or LSM $\geq 14.6 \mathrm{kPa}$. Liver fibrosis stage was measured at baseline and at each subsequent visit in order to determine the liver fibrosis stage at each visit and, hence, the degree of liver fibrosis progression. A liver fibrosis stage of at least one stage higher with respect to the baseline was considered liver fibrosis progression. Liver stiffness measurement was planned for each study visit.

In cirrhotic patients, liver decompensation was considered as the following hepatic events: portal hypertensive gastrointestinal bleeding (PHGB), ascites, hepatorenal syndrome (HRS), spontaneous bacterial peritonitis (SBP), hepatic encephalopathy (HE), and hepatocarcinoma (HCC).

Health status or cause of death was collected at each visit. Cause of death was classified as AIDS-related, liver-related, or other cause. Exitus due to other causes was specified in the data collection.

HIV and HCV viral loads were obtained using reverse transcription polymerase chain reaction (RT-PCR) (Cobas TaqMan; Roche Diagnostic Systems Inc., Pleasanton, CA, USA). Undetectable HIV viral load was defined as an HIV RNA viral load of less than 50 copies per mL.

\section{Statistical analysis}

Analysis of the population included in the study was descriptive. Characteristics of patients (qualitative variables) were reported as the number of cases (percentages). Numerical variables were reported as medians (interquartile range). Otherwise, the population description was divided into patients who were receiving HCV treatment during follow-up and those who were not. For patients receiving treatment during follow-up, a comparative analysis according to $\mathrm{HCV}$ genotype, liver fibrosis stage, and previous experience of $\mathrm{HCV}$ therapy was performed in order to identify variables with an impact on the clinical decision to initiate therapy. Those variables were compared using the Chi-square test.

An analysis evaluating the rate of liver fibrosis progression was performed. Liver fibrosis progression was considered as an increase of at least one liver fibrosis stage with respect to the baseline. Patients with liver fibrosis stage F4 at baseline were censored from analysis. The mortality rate was calculated as the overall number of deaths in the total population. Otherwise, mortality rates were determined for patients with cirrhosis (F4) and F0-F3 patients, specifying cause of death.

\section{Ethics}

This study was conducted according to the principles of Good Clinical Practice (Ministry of Health, Royal Decree 223/2004 of 6 February) and the Declaration of Helsinki. The study did not require informed consent because the patients were not directly interviewed and completely anonymous information was collected from existing records, ensuring the protection of personal data in accordance with Law 15/1999 of 13 December on Personal Data Protection. The study coordinator presented the study protocol (protocol code: FIBICO-0015/01017-2015) to the Coordinating Ethics Committee for Biomedical Research in Andalusia for evaluation and obtained approval (2402819-05/15). Those responsible for the provision of health services where the study occurred were given a copy of the protocol and documents evidencing approval by the Ethics Committee in accordance with the procedures and legal requirements. 


\section{Results}

\section{Baseline population and characteristics}

A total of $3474 \mathrm{HIV} / \mathrm{HCV}$ co-infected patients were included in the cohort and formed the study population. This implies a prevalence of active HCV infection in the south of Spain of $22.4 \%$. The distribution of patients according to HCV genotype was: $1944 \mathrm{HCV}$ genotype 1 (56.07 \%), $38 \mathrm{HCV}$ genotype $2(1.01 \%), 559 \mathrm{HCV}$ genotype 3 (16.08\%), $736 \mathrm{HCV}$ genotype $4(21.2 \%)$, three HCV genotype $6(0.08 \%)$, and 194 whose HCV genotype was not available (5.56 \%). Among patients infected by HCV genotype 1, 862 were genotype 1a (44.2\%), 461 genotype $1 \mathrm{~b}(23.6 \%), 49 \mathrm{HCV}$ genotype $1 \mathrm{ab}(2.6 \%)$, and in 572 patients, the HCV subtype was not determined $(29.6 \%)$. When patients were sorted according to liver fibrosis stage, 1152 patients were F0-F1 (33.2 \%), 513 were F2 (14.4\%), 641 were F3 (18.2\%), 761 were F4 $(21.9 \%)$, and in 407 patients, liver fibrosis was not staged $(12.3 \%)$. When patients were grouped according to previous experience of HCV therapy, 2453 were naïve (70.6\%), 898 did not respond to pegylated interferon plus ribavirin (Peg-IFN/RBV) (25.8\%), and 123 did not respond to Peg-IFN/RBV in combination with boceprevir or telaprevir $(3.6 \%)$.

\section{Follow-up: clinical events}

After 6 months of follow-up, liver fibrosis stage had progressed by at least one stage in 248 patients [7.1\%; $95 \%$ confidence interval (CI): 6.3-8 \%]. Of these, $108 \mathrm{F0}$ F1 patients progressed to F2-F4 (9.3\%; $95 \%$ CI: $7.8-$ $11.2 \%), 80 \mathrm{~F} 2$ patients progressed to F3-F4 (15.6\%; $95 \%$ CI: $12.7-19 \%)$, and $59 \mathrm{~F} 3$ patients progressed to $\mathrm{F} 4(9.2 \%$; $95 \%$ CI: 7.2-11.7\%).

Among 761 patients with cirrhosis, 52 (6.8\%; $95 \%$ CI: 5.2-8.9\%) developed hepatic decompensation during the 6 months of follow-up, which included ascites in 28 $(53.8 \%)$ patients, $\mathrm{HCC}$ in $14(26.9 \%)$, PHGB in 6 $(11.5 \%)$, HE in $3(5.7 \%)$, and SBP in $1(1.9 \%)$. The cumulative incidence of $\mathrm{HCC}$ in cirrhotic patients during the 6 months of follow-up was $1.8 \%$ (95\% CI: 1.1-3.1\%).

Fifty patients died during follow-up. The mortality rate was $1.4 \%$ (95\% CI: 1.1-1.9\%). Causes of death according to liver fibrosis stage are summarized in Table 2.

\section{Follow-up: treatment implementation}

Eight hundred and nineteen patients $(23.56 \%)$ initiated treatment for HCV infection in the first 6 months of the implementation of a national strategy for the diagnosis, treatment, and management of HCV in Spain, or a rate of 4.55 treatment implementations per day. 2654 patients, therefore, were not treated. The main baseline characteristics of treated and nontreated patients are shown in Table 3 . Of the 52 patients in the decompensated liver stage of F4, 50 initiated therapy against $\mathrm{HCV}$ infection. The two patients who did not initiate therapy died before starting treatment due to complications of liver decompensation.

The proportion of treated patients by liver fibrosis stage, $\mathrm{HCV}$ genotype, and previous experience of HCV therapy are shown in Fig. 1. A total of $90 \mathrm{~F} 0-\mathrm{F} 1$ patients initiated treatment during the follow-up. Of these, 49 (54.4\%) experienced treatment failure with a DAA + Peg-IFN/RBV regimen, 27 $(28.8 \%)$ showed extra-hepatic manifestations or another comorbidity, $13(14.4 \%)$ were treated for epidemiological reasons, and $1(2.4 \%)$ was a renal transplant patient.

\section{Discussion}

We report the first results of the impact of the Spanish national strategic plan for the diagnosis, treatment, and management of $\mathrm{HCV}$ on $\mathrm{HIV} / \mathrm{HCV}$ co-infected patients in the HERACLES cohort. During the first 6 months of follow-up, a high proportion $(819,23.56 \%)$ of HIV/HCV co-infected patients included in the cohort initiated DAA-based anti-HCV therapy. However, despite this, there was a high incidence of negative short-term outcomes among the HIV/HCV patients in our cohort; in this regard, $248(7.1 \%$; $95 \%$ CI: 6.3-8\%) patients progressed by at least one stage of liver fibrosis, 52 cirrhotic patients $(6.8 \%$; $95 \%$ CI: 5.2-8.9\%) developed hepatic decompensation, and 50 patients $(1.4 \%$; $95 \%$ CI: $1.1-1.9 \%)$ died.

Liver fibrosis stage was the main factor involved in the decision to initiate anti-HCV therapy. Cirrhotic patients constitute the principal sensitive $\mathrm{HCV}$ population group due to the short-term risk of liver decompensation and liver-related death $[10,11]$. The benefit of successful anti-HCV treatment in this subset of patients, in the sense that it significantly reduces both morbidity and mortality, is generally recognized $[3,12]$. The general use of direct-acting antiviral agents is, therefore, a priority for these patients because this is the target population that derives the most benefit in the short term [13]. In our cohort, 761 patients $(24.8 \%)$ showed liver cirrhosis and $392(51.5 \%)$ of these received anti-HCV therapy during the 6 months of follow-up. By contrast, $52(6.8 \%)$ cirrhotic patients suffered liver decompensation during follow-up and 15 (1.9\%) died. On the other hand, the number of active HCV infection patients in our cohort with no or mild liver fibrosis (F0-F2) starting treatment for hepatitis C was low. Stage F0 F1 fibrosis was detected in $1152(33.1 \%)$ patients, and 90 $(7.8 \%)$ of these received anti-HCV therapy. This proportion was significantly lower than for stage F2 (15.3\%), stage F3 (34.6\%), and cirrhotic patients $(51.5 \%)$, most likely due to prioritization policies in the prescription of IFN-free, direct- 
Table 2 Causes of death during follow-up, according to liver fibrosis stage

Table 3 Characteristics of treated and non-treated patients

\begin{tabular}{lllll}
\hline & Overall $(n=3474)$ & $\mathrm{F} 0-\mathrm{F} 3(n=2713)$ & $\mathrm{F} 4(n=761)$ & $p$-Value \\
\hline Death $(n=50)$ & $50(1.4 \%)$ & $34(1.2 \%)$ & $16(2.1 \%)$ & 0.082 \\
Causes & $n=50$ & $n=34$ & $n=16$ & \\
Liver-related & $15(30)$ & $4(11.7 \%)$ & $11(68.7)$ & $<0.001$ \\
Non-AIDS malignancies & $10(19.2)$ & $6(17.6 \%)$ & $4(25)$ & 0.588 \\
Systemic bacterial infection & $7(13.4)$ & $7(20.6 \%)$ & 0 & 0.054 \\
Cardiovascular event & $6(9.6)$ & $6(17.6 \%)$ & 0 & 0.116 \\
Abuse of toxic substances & $4(7.6)$ & $3(8.8 \%)$ & $1(6.3)$ & 0.792 \\
AIDS-related & $3(5.7)$ & $3(8.8 \%)$ & 0 & 0.389 \\
Gastrointestinal bleeding* & $2(3.8)$ & $2(5.9 \%)$ & 0 & 0.771 \\
End-stage renal disease & $2(3.8)$ & $2(5.9 \%)$ & 0 & 0.771 \\
Suicide & $2(3.8)$ & $1(3.1 \%)$ & 0 & 0.66 \\
\hline
\end{tabular}

*Gastrointestinal bleeding does not include bleeding from esophageal varices

\begin{tabular}{|c|c|c|c|}
\hline Characteristics & Treated $(n=819)$ & Non-treated $(N=2654)$ & $p$-Value \\
\hline Age (years), median (IQR) & $50(47-53)$ & $48(45-52)$ & 0.001 \\
\hline \multicolumn{4}{|l|}{ Gender, no. (\%) } \\
\hline Male & $683(83.3)$ & $2437(91.7)$ & \multirow[t]{2}{*}{0.256} \\
\hline Female & $136(16.7)$ & $217(8.3)$ & \\
\hline \multicolumn{4}{|l|}{ Risk group for HCV infection, no. (\%) } \\
\hline IDU & $710(86.7)$ & $2318(87.3)$ & \multirow[t]{3}{*}{0.876} \\
\hline Sexual & $97(11.8)$ & $321(12.1)$ & \\
\hline Blood-derived & $12(1.5)$ & $15(0.6)$ & \\
\hline \multicolumn{4}{|l|}{ HAART, no. (\%) } \\
\hline Receiving & $810(98.9)$ & $2550(96)$ & \multirow[t]{2}{*}{0.214} \\
\hline Non-receiving & $9(1.1)$ & $104(4)$ & \\
\hline CD4+ total count (cells/mL), median (IQR) & $527(344-760)$ & $487(305-702)$ & 0.001 \\
\hline \multicolumn{4}{|l|}{ HCV genotype, no. (\%) } \\
\hline 1 & $508(62.02)$ & $1436(54.2)$ & \multirow[t]{10}{*}{0.72} \\
\hline $1 \mathrm{a}$ & $253(49.8)$ & $609(42.2)$ & \\
\hline $1 b$ & $116(22.8)$ & $345(23.9)$ & \\
\hline $1 \mathrm{a} / \mathrm{b}$ & $16(3.2)$ & $33(2.3)$ & \\
\hline NG & $123(24.2)$ & 449 (31.9) & \\
\hline 2 & $10(1.2)$ & $28(0.9)$ & \\
\hline 3 & $131(15.9)$ & $428(16.1)$ & \\
\hline 4 & $169(20.6)$ & $567(21.3)$ & \\
\hline 6 & $1(0.28)$ & $2(0.2)$ & \\
\hline NG & 0 & $194(7.3)$ & \\
\hline \multicolumn{4}{|l|}{ Liver fibrosis stage, no. (\%) } \\
\hline F0-F1 & $90(10.9)$ & $1062(40)$ & \multirow[t]{5}{*}{$<0.001$} \\
\hline $\mathrm{F} 2$ & $79(9.6)$ & $434(16.3)$ & \\
\hline $\mathrm{F} 3$ & $222(27.1)$ & $419(15.7)$ & \\
\hline $\mathrm{F} 4$ & $392(47.8)$ & $369(13.9)$ & \\
\hline Not staged & $36(4.6)$ & $371(14.1)$ & \\
\hline \multicolumn{4}{|l|}{ Previous experience of HCV therapy, no. (\%) } \\
\hline Naïve & $366(44.6)$ & 2087 (78.6) & \multirow[t]{3}{*}{0.014} \\
\hline Failure of Peg-IFN/RBV & $373(45.5)$ & $525(19.7)$ & \\
\hline Failure of BOC- or TPV-based regimen & $80(9.9)$ & $43(1.7)$ & \\
\hline
\end{tabular}

$I Q R$ interquartile range; IDU injecting drug user; HAART highly active antiretroviral therapy; $N G$ non-genotyped; $B O C$ boceprevir; $T P V$ telaprevir 

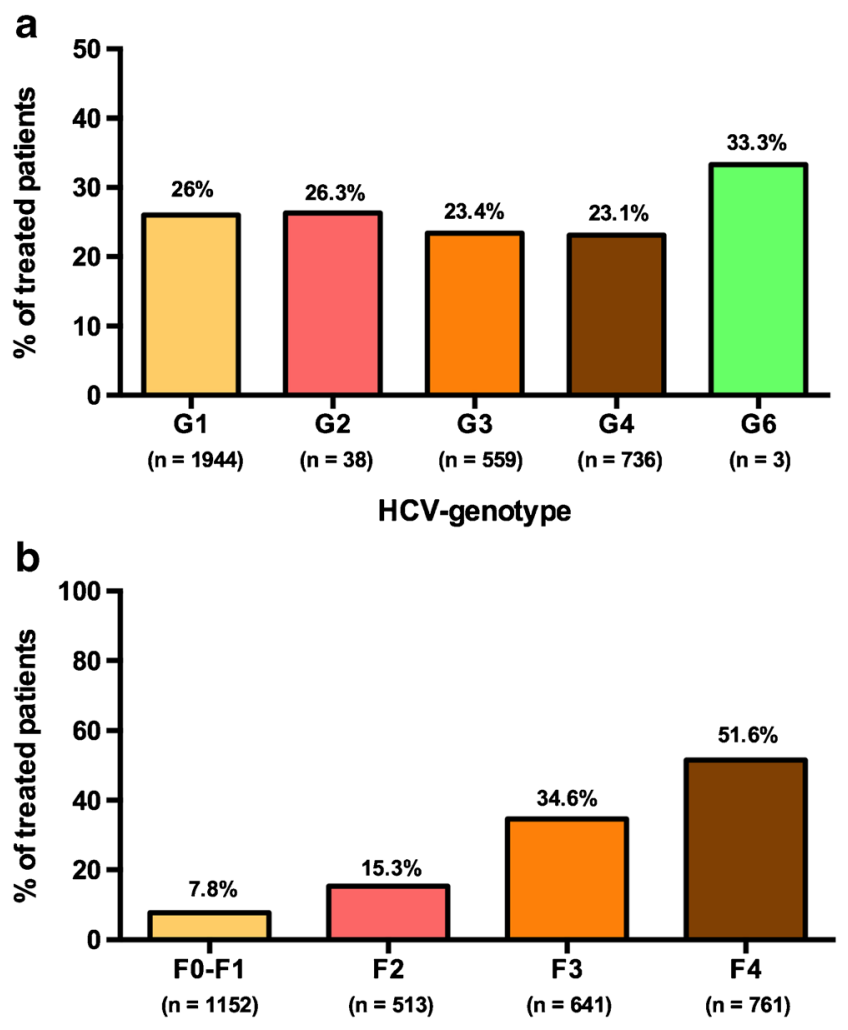

Liver fibrosis stage

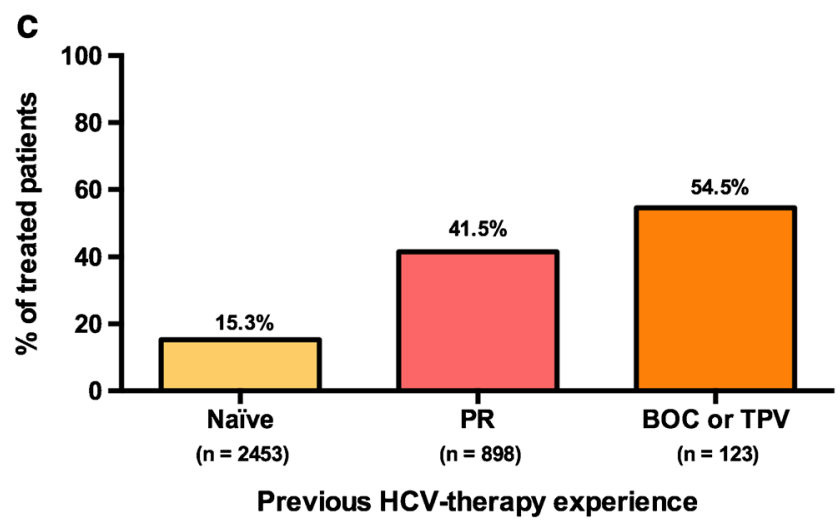

Fig. 1 Distribution of patients receiving hepatitis $\mathrm{C}$ virus (HCV) therapy, according to HCV genotype (a), liver fibrosis stage (b), and previous experience of $\mathrm{HCV}$ therapy (c), with respect to the global population

acting antiviral agent-based therapies for HCV in the Spanish national plan. In addition, HIV/HCV co-infected patients represent a highly sensitive population due to the high rate of liver fibrosis progression compared to $\mathrm{HCV}$ monoinfected patients $[14,15]$. Indeed, in our cohort, $8 \%$ of F0-F2 patients progressed to the F3-F4 stage of liver fibrosis during just 6 months of follow-up. This observation may be added to the list of reasons in favor of prioritizing anti-HCV treatment, regardless of fibrosis stage, in patients with $\mathrm{HIV} / \mathrm{HCV}$ coinfection.

Chronic HCV infection increases the mortality rate for hepatic and extra-hepatic diseases [16]. In our cohort, 52
(1.5\%) patients died during the 6 months of follow-up. Liver-related deaths accounted for $30 \%$ of all causes of death in our study. The course of chronic HCV is accelerated in HIV/HCV-co-infected patients, with more rapid liver fibrosis progression and more frequent hepatic decompensation events compared to HCV monoinfected patients [7, 8, 17]. Consequently, HCV-related liver complications have emerged as a significant cause of mortality among HIV/HCV co-infected patients. HAART is an important factor in slowing liver fibrosis progression, although its use is not sufficient to reduce the rates of endstage liver disease to those of HCV monoinfected patients [7]. For this reason, HIV co-infected patients are considered a priority for receiving anti-HCV therapy regardless of liver fibrosis stage, so as to reduce the rate of hepatic decompensation. As expected in a cohort of HIV-infected patients containing a high proportion of subjects with suppressed HIV viral loads due to HAART, mortality due to AIDS-related events was low, accounting for $5.7 \%$ of causes of death. In contrast, non-AIDS/non-liver-related conditions constituted the main cause of death in our cohort. Cardiovascular diseases and non-AIDS/non-liver malignancies have been related to both HCV and HIV infection. In our cohort, non-AIDS/non-liver malignancies comprised $64.3 \%$ of all causes of death. The incidence of nonAIDS-defining malignancies is elevated in chronic HIV infection $[18,19]$. In addition, some evidence has suggested that HCV may be associated with the development of malignancies such as B-cell non-Hodgkin lymphoma, intrahepatic cholangiocarcinoma, and the incidence of pancreatic cancer [20]. Genomic and/or HCV replicative sequences have been detected in extra-hepatic localizations and it has been speculated that HCV may promote persistent inflammation in extra-hepatic organs and induce cancerous transformations [20]. Finally, in our cohort, five patients died due to cardiovascular disease; in other words, $9.6 \%$ of all deaths. This is not striking, because cardiovascular disease is a major cause of morbidity and mortality in HIV-infected patients, accounting for at least $10 \%$ of deaths [21,22], and the evidence in favor of HCV infection promoting major risk factors for cardiovascular disease is compelling [23].

The prevalence of HCV active infection among HIVinfected patients has decreased in recent years. The prevalence of chronic HCV infection in Spain in 2002 was $54 \%$ [24], decreasing to $34 \%$ in 2009 [25], then to $22.1 \%$ (402 out of 1882) in 2015 [26]. The baseline prevalence (March 2015) of chronic HCV infection in our cohort was similar, at $22.4 \%$. The progressive decrease of active $\mathrm{HCV}$ infection in our setting is due to at least three factors. Firstly, to changes in the route of HIV transmission. The decline of injecting drug users (IDUs) as the predominant route for transmission of HIV infection is probably the 
main factor governing the steady decrease in active HCV infection in Spain [27]. The prevalence of IDUs among Spanish patients newly infected with HIV decreased from $67.3 \%$ in 1997 to $14.5 \%$ in 2006, with a parallel reduction in the rate of HCV co-infection (from $73.8 \%$ in 1997 to $19.8 \%$ in 2006) in these patients [27]. Secondly, the reduction in active $\mathrm{HCV}$ infection is due to the high mortality among $\mathrm{HIV} / \mathrm{HCV}$ co-infected patients. It is well established that individuals with $\mathrm{HIV} / \mathrm{HCV}$ co-infection have significantly higher morbidity and mortality rates compared to individuals with HIV monoinfection [5, 28, 29]. As a result, liver-related complications currently represent one of the leading causes of death in the HIVinfected population. This particular aspect may have contributed to the progressive decrease in active $\mathrm{HCV}$ infection among HIV-infected patients in our area. Finally, the third reason is the use of highly effective anti-HCV therapy. The great majority of clinical trials evaluating the efficacy of DAAs have demonstrated high SVR rates in both $\mathrm{HIV} / \mathrm{HCV}$ co-infected and HCV-infected patients $[1,2]$. In our cohort, during the first 6 months of implementation of this national strategic plan for HCV, 819 (23.5\%) of the $\mathrm{HIV} /$ active HCV-infected patients received DAA-based anti-HCV therapy. It is assumed that, in the coming months, the widespread use of anti-HCV therapy will increase HCV cure rates and, hence, reduce active HCV infection rates among HIV/HCV co-infected individuals.

Our study presents several limitations that should be noted. Firstly, in the initiation phase of the study, not all DAA regimens had been approved in Spain and, so, the impact of this national strategic plan for the diagnosis, treatment, and management of HCV may be limited in this respect. Secondly, due to the fact that only HIV/HCV co-infected patients were included in our cohort, it was not possible to compare the impact of implementation on HCV monoinfected patients. Thirdly, liver fibrosis stage could not be established in $12.3 \%$ of patients included in the study. These patients represent a special population for whom the national strategic plan cannot be implemented because of the absence of priority markers. Finally, although we mentioned that the majority $(99.3 \%)$ of $\mathrm{HIV} / \mathrm{HCV}$ co-infected patients being followed in our area were included in our cohort, we do not know what proportion of patients have no access to clinical care.

In conclusion, during the first 6 months, 1 in $4 \mathrm{HIV} / \mathrm{HCV}$ co-infected patients, mainly with advanced liver fibrosis, were treated in the HERACLES cohort as a result of the Spanish national strategic plan for the diagnosis, treatment, and management of HCV. However, despite this apparent good news, the high incidence of negative short-term outcomes observed in our cohort makes it indispensable to continue providing extra resources to increase the rate of $\mathrm{HIV} / \mathrm{HCV}$ co-infected patients receiving anti-HCV therapy, in order to improve the prognosis for this highly sensitive population.
Acknowledgments We acknowledge Ana Gordon, Patricia Monje, and María Isabel Mayorga for their support in the data collection.

\section{Compliance with ethical standards}

Funding This work was supported by the Ministerio de Sanidad (RD12/0017/0012 and PI15/01017) integrated in the Plan Nacional de $\mathrm{I}+\mathrm{D}+\mathrm{I}$ and cofinanced by the ISCIII-Subdirección General de Evaluación and the Fondo Europeo de Desarrollo Regional (FEDER). A.R.-J. was the recipient of a Post-Doctoral Research Extension Grant from the Fundación Progreso y Salud (0024-RH-2013 Consejería de Salud, Innovación y Ciencia de la Junta de Andalucia). The HEPAVIR group was the recipient of a Research Network Support Grant from the Fundación Progreso y Salud (Consejería de Salud, Innovación y Ciencia de la Junta de Andalucia; AC-0095-2013, AYUDAS A GRUPOS DE INVESTIGACION).

Conflict of interest We declare no competing interests. The authors or their institutions have not received at any time payment or services from a third party for any aspect of the submitted work (data monitoring board, study design, manuscript preparation, statistical analysis, etc.).

Ethical approval The study coordinator presented the study protocol (protocol code: FIBICO-0015/01017-2015) to the Coordinating Ethics Committee for Biomedical Research in Andalusia for evaluation and obtained approval (240-2819-05/15).

Informed consent This study does not need informed consent.

Contributions Dr. Rivero had full access to all of the data in the study and takes responsibility for the integrity of the data and the accuracy of the data analysis.

Study concept and design: Rivero-Juarez, Lopez-Cortes, Rivero.

Administrative, technical, or material support: all authors.

Analysis and interpretation of the data: Rivero-Juarez, Rivero.

Drafting of the manuscript: Rivero-Juarez, Rivero.

Critical revision of the manuscript for important intellectual content: all authors.

Statistical analysis: Rivero-Juarez, Rivero.

Obtained funding: Rivero.

Study supervision: Rivero.

Open Access This article is distributed under the terms of the Creative Commons Attribution 4.0 International License (http:// creativecommons.org/licenses/by/4.0/), which permits unrestricted use, distribution, and reproduction in any medium, provided you give appropriate credit to the original author(s) and the source, provide a link to the Creative Commons license, and indicate if changes were made.

\section{References}

1. Recommendations for Testing, Managing, and Treating Hepatitis $\mathrm{C}$ of the American Association for the Study of Liver Diseases (AASLD) and the Infectious Diseases Society of America (IDSA). Available online at: http://www.hcvguidelines.org. Last accessed June 5th 2016

2. European Association for the Study of the Liver (EASL) Recommendations on Treatment of Hepatitis C 2015. Available online at: http://www.easl.eu/research/our-contributions/clinicalpractice-guidelines/detail/recommendations-on-treatment-ofhepatitis-c-2015. Last accessed June 5th 2016 
3. Mira JA, Rivero-Juárez A, López-Cortés LF et al (2013) Benefits from sustained virologic response to pegylated interferon plus ribavirin in $\mathrm{HIV} /$ hepatitis $\mathrm{C}$ virus-coinfected patients with compensated cirrhosis. Clin Infect Dis 56:1646-1653

4. Plan estratégico para el abordaje de la Hepatitis c en el Sistema Nacional de Salud. Available online at: http://www.msssi.gob. es/ciudadanos/enfLesiones/enfTransmisibles/docs/plan estrategico hepatitis_C.pdf. Last accessed June 5th 2016

5. Branch AD, Van Natta ML, Vachon ML et al (2012) Mortality in hepatitis C virus-infected patients with a diagnosis of AIDS in the era of combination antiretroviral therapy. Clin Infect Dis 55:137144

6. Neukam K, Suarez Santamaría M, Rivero-Juárez A et al (2016) HIV coinfection impairs the response to DAA-based HCV therapy. The International Liver Congress, Barcelona, Spain, April 13-17 2016. Abstract LB-P513

7. Lo Re V 3rd, Kallan MJ, Tate JP et al (2014) Hepatic decompensation in antiretroviral-treated patients co-infected with HIV and hepatitis $\mathrm{C}$ virus compared with hepatitis $\mathrm{C}$ virus-monoinfected patients: a cohort study. Ann Intern Med 160:369-379

8. Pineda JA, García-García JA, Aguilar-Guisado M et al (2007) Clinical progression of hepatitis $\mathrm{C}$ virus-related chronic liver disease in human immunodeficiency virus-infected patients undergoing highly active antiretroviral therapy. Hepatology 46:622-630

9. Rivero-Juarez A, Gutierrez-Valencia A, Castaño M et al (2015) Dimension of chronic hepatitis $\mathrm{C}$ virus in HIV-infected patients in the interferon-free era: an overview from south Spain. Eur J Clin Microbiol Infect Dis 34:2247-2255

10. Merchante N, Girón-González JA, González-Serrano M et al (2006) Survival and prognostic factors of HIV-infected patients with HCV-related end-stage liver disease. AIDS 20:49-57

11. Macías J, Márquez M, Téllez F et al (2013) Risk of liver decompensation among HIV/hepatitis $\mathrm{C}$ virus-coinfected individuals with advanced fibrosis: implications for the timing of therapy. Clin Infect Dis $57: 1401-1408$

12. van der Meer AJ, Veldt BJ, Feld JJ et al (2012) Association between sustained virological response and all-cause mortality among patients with chronic hepatitis $\mathrm{C}$ and advanced hepatic fibrosis. JAMA 308:2584-2593

13. Martin NK, Vickerman P, Dore GJ et al (2016) Prioritization of $\mathrm{HCV}$ treatment in the direct-acting antiviral era: An economic evaluation. J Hepatol 65:17-25

14. Macías J, Berenguer J, Japón MA et al (2009) Fast fibrosis progression between repeated liver biopsies in patients coinfected with human immunodeficiency virus/hepatitis $\mathrm{C}$ virus. Hepatology 50: 1056-1063

15. Chen JY, Feeney ER, Chung RT (2014) HCV and HIV co-infection: mechanisms and management. Nat Rev Gastroenterol Hepatol 11: $362-371$
16. Lee MH, Yang HI, Lu SN et al (2012) Chronic hepatitis C virus infection increases mortality from hepatic and extrahepatic diseases: a community-based long-term prospective study. J Infect Dis 206:469-477

17. Sulkowski MS, Mehta SH, Torbenson MS et al (2007) Rapid fibrosis progression among HIV/hepatitis C virus-co-infected adults. AIDS 21:2209-2216

18. Silverberg MJ, Chao C, Leyden WA et al (2009) HIV infection and the risk of cancers with and without a known infectious cause. AIDS 23:2337-2345

19. Grulich AE, van Leeuwen MT, Falster MO et al (2007) Incidence of cancers in people with HIV/AIDS compared with immunosuppressed transplant recipients: a meta-analysis. Lancet 370:59-67

20. Fiorino S, Bacchi-Reggiani L, de Biase D et al (2015) Possible association between hepatitis $\mathrm{C}$ virus and malignancies different from hepatocellular carcinoma: A systematic review. World J Gastroenterol 21:12896-12953

21. Data Collection on Adverse Events of Anti-HIV drugs (D:A:D) Study Group, Smith C, Sabin CA et al (2010) Factors associated with specific causes of death amongst HIV-positive individuals in the D:A:D Study. AIDS 24:1537-1548

22. Mocroft A, Reiss P, Gasiorowski J et al (2010) Serious fatal and nonfatal non-AIDS-defining illnesses in Europe. J Acquir Immune Defic Syndr 55:262-270

23. Katsi V, Felekos I, Skevofilax S et al (2015) Cardiovascular disease and hepatitis $\mathrm{C}$ virus infection: an irrelevant statement or a hot relationship? Cardiol Rev 23:11-17

24. González-García JJ, Mahillo B, Hernández S et al (2005) Prevalences of hepatitis virus coinfection and indications for chronic hepatitis $\mathrm{C}$ virus treatment and liver transplantation in Spanish HIV-infected patients. The GESIDA 29/02 and FIPSE 12185/01 Multicenter Study. Enferm Infecc Microbiol Clin 23:340-348

25. Serrano-Villar S, Sobrino-Vegas P, Monge S et al (2015) Decreasing prevalence of $\mathrm{HCV}$ coinfection in all risk groups for HIV infection between 2004 and 2011 in Spain. J Viral Hepat 22: 496-503

26. Berenguer J, Rivero A, Jarrín I et al (2016) Human immunodeficiency virus/hepatitis $C$ virus coinfection in Spain: prevalence and patient characteristics. Open Forum Infect Dis 3:ofw059

27. Pérez Cachafeiro S, Del Amo J, Iribarren JA et al (2009) Decrease in serial prevalence of coinfection with hepatitis $\mathrm{C}$ virus among HIV-infected patients in Spain, 1997-2006. Clin Infect Dis 48: $1467-1470$

28. Rockstroh JK, Peters L, Grint D et al (2013) Does hepatitis C viremia or genotype predict the risk of mortality in individuals coinfected with HIV? J Hepatol 59:213-220

29. Erqou S, Mohanty A, Murtaza Kasi P et al (2014) Predictors of mortality among United States veterans with human immunodeficiency virus and hepatitis $\mathrm{C}$ virus coinfection. ISRN Gastroenterol 2014:764540 